\title{
The proportion of failures of the Hasse norm principle
}

Article

Accepted Version

Browning, T. D. and Newton, R. (2016) The proportion of failures of the Hasse norm principle. Mathematika, 62 (02). pp. 337-347. ISSN 2041-7942 doi:

https://doi.org/10.1112/S0025579315000261 Available at https://centaur.reading.ac.uk/58164/

It is advisable to refer to the publisher's version if you intend to cite from the work. See Guidance on citing.

To link to this article DOI: http://dx.doi.org/10.1112/S0025579315000261

Publisher: Cambridge University Press

Publisher statement: This version may differ from the version published in Mathematika.

All outputs in CentAUR are protected by Intellectual Property Rights law, including copyright law. Copyright and IPR is retained by the creators or other copyright holders. Terms and conditions for use of this material are defined in the End User Agreement.

www.reading.ac.uk/centaur

\section{CentAUR}

Central Archive at the University of Reading 
Reading's research outputs online 


\title{
THE PROPORTION OF FAILURES OF THE HASSE NORM PRINCIPLE
}

\author{
T.D. BROWNING AND R. NEWTON
}

\begin{abstract}
For any number field we calculate the exact proportion of rational numbers which are everywhere locally a norm but not globally a norm from the number field.
\end{abstract}

\section{INTRODUCTION}

Let $K / k$ be a finite extension of number fields with associated idèle groups $J_{K}$ and $J_{k}$. Let $N_{K / k}: J_{K} \rightarrow J_{k}$ denote the norm map and, in the usual manner, consider the multiplicative groups $K^{*}$ and $k^{*}$ as subgroups of $J_{K}$ and $J_{k}$, respectively. The Hasse norm principle is said to hold for $K / k$ if

$$
k^{*} \cap N_{K / k} J_{K}=N_{K / k} K^{*},
$$

where we view the group $N_{K / k} K^{*}$ of global norms as a subgroup of $k^{*} \cap N_{K / k} J_{K}$, with finite index. When $k=\mathbb{Q}$ the main result of this paper gives the exact proportion of elements in $\mathbb{Q}^{*} \cap N_{K / \mathbb{Q}} J_{K}$ which do not belong to $N_{K / \mathbb{Q}} K^{*}$.

The classical Hasse norm theorem states that an extension $K / k$ satisfies the Hasse norm principle when it is cyclic. For non-cyclic extensions there can be counter-examples. The most elementary example is provided by the biquadratic extension $\mathbb{Q}(\sqrt{13}, \sqrt{17}) / \mathbb{Q}$, with $5^{2}$ being represented everywhere locally by norms but not globally. Subsequently, a vast literature has emerged around the Hasse norm principle, resulting in a long list of explicit conditions under which one can conclude that a given extension $K / k$ satisfies the Hasse norm principle. For example, the Hasse norm principle holds if:

- the degree $[K: k]$ is prime (Bartels [Bar81a]); or

- the Galois group of $N / k$ is dihedral of order $2[K: k]$, where $N$ is the normal closure of $K$ over $k$ (Bartels [Bar81b]); or

- $K / k$ is Galois and every Sylow subgroup of the Galois group is cyclic (Gurak [Gur78, Cor. 3.2]).

The knot group $\mathfrak{K}(K / k)$ of the extension $K / k$ is defined to be the finite quotient group $\left(k^{*} \cap N_{K / k} J_{K}\right) / N_{K / k} K^{*}$ and the knot number $i(K / k)$ is the cardinality of this group. The Hasse norm principle fails for $K / k$ if and only if $i(K / k)>1$.

Date: October 7, 2015.

2010 Mathematics Subject Classification. 11G35 (11R37, 12G05, 14G05). 
The calculation of knot groups can be very difficult in practice. Razar Raz77] has provided a general method for computing $i(K / k)$ via the construction of large abelian extensions of $K$. For bicyclic extensions the situation is simpler. Although we shall see that the following fact is a simple consequence of known results, we state it here for ease of reference.

Theorem 1.1. Let $K / k$ be a Galois extension of number fields with Galois group $\mathbb{Z} / m \mathbb{Z} \times \mathbb{Z} / n \mathbb{Z}$. For a place $v$ of $k$, let $g_{v}$ be the number of places of $K$ above $v$. Let $g$ be the greatest common divisor of all the $g_{v}$. Then $\mathfrak{K}(K / k) \cong \mathbb{Z} / g \mathbb{Z}$.

An alternative point of view comes from observing that the failure of the Hasse norm principle for $K / k$ is equivalent to the failure of the Hasse principle for the affine variety

$$
V: \quad N_{K / k}(\Xi)=c,
$$

for an element $c \in k^{*}$. This equation defines a principal homogeneous space for the norm one $k$-torus $T=R_{K / k}^{1} \mathbb{G}_{m}$ and its associated Tate-Shafarevich group is isomorphic to the knot group $\mathfrak{K}(K / k)$. Work of Sansuc San81] implies that the failure of the Hasse principle on any smooth projective model of $V$ is controlled by the Brauer-Manin obstruction. Following Manin [Man71, one can therefore construct the unramified Brauer group of $V$, which is defined to be $\operatorname{Br}\left(V^{c}\right)=H_{\text {ét }}^{2}\left(V^{c}, \mathbb{G}_{m}\right)$ for any smooth compactification $V^{c}$ of $V$, together with a Brauer set $V^{c}\left(\mathbf{A}_{k}\right)^{\mathrm{Br}} \subseteq V^{c}\left(\mathbf{A}_{k}\right)$ which contains the $k$-rational points of $V^{c}$ and so potentially obstructs the existence of rational points

In principle, both the knot group and the Brauer-Manin obstruction can produce a decision algorithm for the existence of $k$-rational points on a particular variety $V$ given by (1.1). Specialising to biquadratic extensions $K / k$, Hürlimann [Hür86] has constructed an explicit isomorphism

$$
\psi: k^{*} / \prod_{i=1}^{3} N_{K_{i} / k} K_{i}^{*} \stackrel{\sim}{\longrightarrow} \mathfrak{K}(K / k), \quad \psi(t)=t^{2},
$$

where $K_{1}, K_{2}, K_{3}$ are the three quadratic subfields of $K$, which he has used to produce criteria for the solubility of (1.1). More recently, Wei [Wei14, §2] has used the Brauer-Manin obstruction to do the same thing for biquadratic extensions $K / k$ with $k=\mathbb{Q}$.

Returning to the general case, let $K / k$ be an extension of number fields with $i(K / k)>1$. In practice it can be very challenging to determine whether or not a given element $c \in k^{*} \cap N_{K / k} J_{K}$ fails to belong to $N_{K / k} K^{*}$, or equivalently, whether or not $c \in k^{*}$ produces a counter-example to the Hasse principle for the affine variety $V$ in (1.1). In order to compute the Brauer-Manin obstruction one needs to construct the unramified Brauer group. While it is relatively easy to calculate $\operatorname{Br}\left(V^{c}\right) / \operatorname{Br}(k)$ (cf. [CTK98]), finding explicit generators that lift to 
elements of $\operatorname{Br}\left(V^{c}\right)$ entails considerable strain. Assuming that $K / k$ is Galois, Wei [Wei14] has transformed this problem into one that involves constructing a special abelian extension of $K$, but so far this plan has only been executed for biquadratic (and certain cyclotomic) extensions of $\mathbb{Q}$.

The primary aim of this paper is to measure the density of counter-examples to the Hasse principle for $V$ for a general number field $K / \mathbb{Q}$. In doing so, we will succeed in proving asymptotic formulae for each of the counting functions

$$
\begin{aligned}
N_{\text {loc }}(B) & =\#\left\{t \in \mathbb{Q}^{*} \cap N_{K / \mathbb{Q}} J_{K}: H(t) \leqslant B\right\}, \\
N_{\text {glob }}(B) & =\#\left\{t \in N_{K / \mathbb{Q}} K^{*}: H(t) \leqslant B\right\}, \\
N_{\text {ce }}(B) & =N_{\text {loc }}(B)-N_{\text {glob }}(B),
\end{aligned}
$$

where $H(t)=\max \{|a|,|b|\}$ if $t=a / b$ for coprime integers $a, b$. This will allow us to draw the following conclusion.

Theorem 1.2. For any number field $K / \mathbb{Q}$ we have

$$
\lim _{B \rightarrow \infty} \frac{N_{\mathrm{ce}}(B)}{N_{\mathrm{loc}}(B)}=1-\frac{1}{i(K / \mathbb{Q})} .
$$

The proof of this result avoids having to construct the unramified Brauer group explicitly and instead uses the knot group in an essential way. A consequence of our result is that a positive proportion of elements of $\mathbb{Q}^{*} \cap N_{K / \mathbb{Q}} J_{K}$ give counter-examples to the Hasse principle for $V$ unless, of course, the knot group is trivial. For Galois extensions $K / \mathbb{Q}$ with Galois group $\mathbb{Z} / p \mathbb{Z} \times \mathbb{Z} / p \mathbb{Z}$, for example, Theorem 1.1 shows that the proportion is $1-\frac{1}{p}$ in Theorem 1.2 provided that there is no place with local degree $p^{2}$.

It is interesting to compare Theorem 1.2 with recent work of Bhargava Bha14, which shows that a positive proportion of plane cubic curves over $\mathbb{Z}$ that are everywhere locally soluble actually produce counter-examples to the Hasse principle. In [Bha14, Conj. 6], the precise proportion is conjectured to be $1-\frac{1}{3}$, something that bears comparison with our own discovery.

It is now time to reveal our asymptotic formulae for $N_{\text {loc }}(B)$ and $N_{\text {glob }}(B)$. Let $\mathscr{P}_{K}$ be the set of unramified rational primes $p$ for which $\operatorname{gcd}\left(f_{1}, \ldots, f_{r}\right)=1$ whenever $(p)$ factorises in $\mathfrak{o}_{K}$ as $\mathfrak{p}_{1} \ldots \mathfrak{p}_{r}$, for prime ideals $\mathfrak{p}_{i}$ with $\# \mathfrak{o}_{K} / \mathfrak{p}_{i}=p^{f_{i}}$. According to the Čebotarev density theorem (see [Ser12, §3.2], for example) this set has a natural density and we call this density $\delta_{K}$. The calculation in [Neu91, Cor. 13.6] shows that $\delta_{K} \geqslant 1 /[K: \mathbb{Q}]$, with equality if and only if $K / \mathbb{Q}$ is Galois. Next, we let $I_{K}$ denote the group of non-zero fractional ideals of $K$ and let $N_{K / \mathbb{Q}} I_{K}$ denote the subgroup of $\mathbb{Q}_{>0}$ consisting of norms of fractional ideals. Two finite abelian groups that play a key role in our analysis are

$$
G_{\text {loc }}=\frac{N_{K / \mathbb{Q}} I_{K}}{\mathbb{Q}>0 \cap N_{K / \mathbb{Q}} J_{K}} \quad \text { and } \quad G_{\text {glob }}=\frac{N_{K / \mathbb{Q}} I_{K}}{\mathbb{Q}>0 \cap N_{K / \mathbb{Q}} K^{*}} .
$$


In due course we will show that $\mathbb{Q}_{>0} \cap N_{K / \mathbb{Q}} J_{K}$ is a subgroup of $N_{K / \mathbb{Q}} I_{K}$ and, furthermore, that $\# G_{\text {glob }}=\# G_{\text {loc }} \cdot i(K / \mathbb{Q})$ (see Corollary 2.2 and Lemma 2.3 , respectively). Bearing these definitions in mind, we may now record the following result.

Theorem 1.3. For any number field $K / \mathbb{Q}$ there exists a constant $c(K)>0$ such that

$$
N_{\mathrm{loc}}(B)=\frac{1}{\# G_{\mathrm{loc}}} \frac{c(K) B^{2}}{(\log B)^{2\left(1-\delta_{K}\right)}}(1+o(1))
$$

and

$$
N_{\text {glob }}(B)=\frac{1}{\# G_{\text {glob }}} \frac{c(K) B^{2}}{(\log B)^{2\left(1-\delta_{K}\right)}}(1+o(1)) .
$$

Recalling that $N_{\text {ce }}(B)=N_{\text {loc }}(B)-N_{\text {glob }}(B)$, the statement of Theorem 1.2 is an immediate consequence of this result.

Loughran Lou14 has developed some far-reaching conjectures about counting the number of varieties in a family which contain points everywhere locally. We may compactify the equation $N_{K / \mathbb{Q}}(\Xi)=c$ in order to view it as a family of varieties over $\mathbb{P}^{1}$. One finds that there are two singular fibres over 0 and $\infty$, both of which are non-split. Thus the asymptotic formula for $N_{\text {loc }}(B)$ in Theorem 1.3 is consistent with the predictions in [Lou14, §1.3].

Our analysis of $N_{\text {loc }}(B)$ and $N_{\text {glob }}(B)$ is based on the Dirichlet series approach of Odoni Odo73. Using properties of Artin $L$-functions, Odoni succeeded in getting an asymptotic formula for the number of positive integers in an interval which belong to $N_{K / \mathbb{Q}} K^{*}$. We will show that his argument can be extended to handle the counting functions $N_{\text {loc }}(B)$ and $N_{\text {glob }}(B)$.

Returning once more to biquadratic extensions $K=\mathbb{Q}(\sqrt{a}, \sqrt{b})$ with knot number $i(K / \mathbb{Q})>1$, consider the 5 -dimensional affine hypersurface

$$
\left(x^{2}-a y^{2}\right)\left(z^{2}-b t^{2}\right)\left(u^{2}-a b w^{2}\right)=d,
$$

for $d \in \mathbb{Q}^{*}$. A trivial modification of related work due to de la Bretèche and Browning dlBB14 would guarantee counter-examples to the Hasse principle for this equation for a positive proportion of $d \in \mathbb{Q}^{*}$, which is in contrast to the asymptotic formula for $N_{\text {ce }}(B)$ provided by Theorem 1.3 . It follows from (1.2) that $c=d^{2}$ is a counter-example to the Hasse principle for $V$ whenever $d \in \mathbb{Q}^{*}$ produces a counter-example to the Hasse principle for (1.4). In fact, many of the known failures of the Hasse principle for $V$ (see [CF67, Exercise 5]) involve the square of rational numbers and it is tempting to suppose that the set of rational numbers producing counter-examples to the Hasse principle is very sparse. Our work shows that this set has density $\frac{1}{2}$ in $\mathbb{Q}^{*} \cap N_{K / \mathbb{Q}} J_{K}$ and density zero in $\mathbb{Q}^{*}$, but that it is not nearly as sparse as the set of squares. 
For biquadratic extensions $K / \mathbb{Q}$ we have $\delta_{K}=1 / 4$ in Theorem 1.3 and it follows from Theorem 1.1 that $\mathfrak{K}(K / \mathbb{Q}) \cong \mathbb{Z} / 2 \mathbb{Z}$ when $i(K / \mathbb{Q})>1$. In the special case $K=\mathbb{Q}(\sqrt{13}, \sqrt{17})$ an inspection of [Wei14, Example 10] shows that $\mathfrak{K}(K / \mathbb{Q})$ is generated by -1 . This easily implies that $N_{\text {glob }}(B)=\frac{1}{2} N_{\text {loc }}(B)$, an observation which affords the following especially succinct result.

Theorem 1.4. Let $K=\mathbb{Q}(\sqrt{13}, \sqrt{17})$. Then there exists $c>0$ such that

$$
N_{\mathrm{ce}}(B)=\frac{1}{2} N_{\mathrm{loc}}(B) \sim \frac{c B^{2}}{(\log B)^{3 / 2}} .
$$

We close the introduction by summarising the contents of the paper. In $\$ 2$ we shall establish Theorem 1.1 and discuss the group $N_{K / \mathbb{Q}} I_{K}$ of norms of fractional ideals belonging to $\bar{K}$, in preparation for the proof of Theorem 1.3 . Finally in $\$ 3$ we shall prove this result.

Acknowledgements. While working on this paper the first author was supported by ERC grant 306457. The authors are grateful to Jean-Louis ColliotThélène, Daniel Loughran and Damaris Schindler for comments on an earlier version of this paper, and to Hendrik Lenstra and Bart de Smit for pointing out some useful references.

\section{Norm GROUPS AND CLASS FIELD THEORY}

We begin by proving Theorem 1.1. Let $K / k$ be a Galois extension with Galois group $\mathbb{Z} / m \mathbb{Z} \times \mathbb{Z} / n \mathbb{Z}$. By Hürlimann [Hür84, Cor. 2.10], we have $\mathfrak{K}(K / k) \cong H^{3}\left(G, K^{*}\right)$ for a bicyclic extension. Let $C_{K}=J_{K} / K^{*}$ denote the idèle class group of $K$. We have $H^{3}\left(G, J_{K}\right)=1$ (see [NSW08, Cor. 8.1.3], for example). Taking Galois cohomology of the short exact sequence

$$
1 \rightarrow K^{*} \rightarrow J_{K} \rightarrow C_{K} \rightarrow 1
$$

therefore gives

$$
\cdots \rightarrow H^{2}\left(G, J_{K}\right) \rightarrow H^{2}\left(G, C_{K}\right) \rightarrow H^{3}\left(G, K^{*}\right) \rightarrow 1 .
$$

By [HN52, Thm. 2.7], the image of $H^{2}\left(G, C_{K}\right)$ in $H^{3}\left(G, K^{*}\right)$ is a cyclic group of order $g$, where $g$ is as in the statement of Theorem 1.1. Since (2.1) is exact, this completes the proof of Theorem 1.1.

Returning to general extensions $K / \mathbb{Q}$, in preparation for our analysis of $N_{\text {loc }}(B)$ and $N_{\text {glob }}(B)$ we collect some facts about the group $N_{K / \mathbb{Q}} I_{K}$ of norms of fractional ideals of $K$ and its subgroups. The following result is well known.

Lemma 2.1. Let $\alpha \in \mathbb{Q}$. Then $\alpha \in N_{K / \mathbb{Q}} I_{K}$ if and only if $\alpha>0$ and for every prime number $p$ the greatest common divisor of the residue degrees $f_{\mathfrak{p} / p}$ of the primes above $p$ divides $\operatorname{ord}_{p}(\alpha)$. 
Proof. Let $\mathfrak{a} \in I_{K}$. Then

$$
N_{K / \mathbb{Q}}(\mathfrak{a})=\prod_{p} p^{\sum_{\mathfrak{p} \mid p} f_{\mathfrak{p} / p} \operatorname{ord}_{\mathfrak{p}}(\mathfrak{a})}
$$

where the product runs over all prime numbers. In particular, the forward implication follows since $\operatorname{ord}_{p}\left(N_{K / \mathbb{Q}}(\mathfrak{a})\right)=\sum_{\mathfrak{p} \mid p} f_{\mathfrak{p} / p} \operatorname{ord}_{\mathfrak{p}}(\mathfrak{a})$. Now let $g_{p}$ be the greatest common divisor of the residue degrees of the primes above $p$. Write $g_{p}=\sum_{\mathfrak{p} \mid p} a_{\mathfrak{p}} f_{\mathfrak{p} / p}$ for $a_{\mathfrak{p}} \in \mathbb{Z}$. Then

$$
p^{g_{p}}=N_{K / \mathbb{Q}}\left(\prod_{\mathfrak{p} \mid p} \mathfrak{p}^{a_{\mathfrak{p}}}\right) .
$$

For $\alpha>0$ we have $\alpha=\prod_{p} p^{\operatorname{ord}_{p}(\alpha)}$, giving the reverse implication.

Corollary 2.2. If $\alpha \in \mathbb{Q}_{>0} \cap N_{K / \mathbb{Q}} J_{K}$, then $\alpha \in N_{K / \mathbb{Q}} I_{K}$.

Proof. Let $p$ be a prime number. Suppose that $\alpha=\prod_{\mathfrak{p} \mid p} N_{K_{\mathfrak{p}} / \mathbb{Q}_{p}}\left(\beta_{\mathfrak{p}}\right)$ with $\beta_{\mathfrak{p}} \in K_{\mathfrak{p}}^{*}$. Then

$$
\operatorname{ord}_{p}(\alpha)=\sum_{\mathfrak{p} \mid p} \operatorname{ord}_{p}\left(N_{K_{\mathfrak{p}} / \mathbb{Q}_{p}}\left(\beta_{\mathfrak{p}}\right)\right)=\sum_{\mathfrak{p} \mid p} f_{\mathfrak{p} / p} \operatorname{ord}_{\mathfrak{p}}\left(\beta_{\mathfrak{p}}\right)
$$

Therefore, the greatest common divisor of the residue degrees $f_{\mathfrak{p} / p}$ divides $\operatorname{ord}_{p}(\alpha)$. Now apply Lemma 2.1.

Following Odoni Odo73, §2], we observe that the norm map induces isomorphisms

$$
\frac{I_{K}}{N_{K / \mathbb{Q}}^{-1}\left(\mathbb{Q}>0 \cap N_{K / \mathbb{Q}} K^{*}\right)} \longrightarrow \frac{N_{K / \mathbb{Q}} I_{K}}{\mathbb{Q}_{>0} \cap N_{K / \mathbb{Q}} K^{*}}=G_{\text {glob }}
$$

and

$$
\frac{I_{K}}{N_{K / \mathbb{Q}}^{-1}\left(\mathbb{Q}_{>0} \cap N_{K / \mathbb{Q}} J_{K}\right)} \longrightarrow \frac{N_{K / \mathbb{Q}} I_{K}}{\mathbb{Q}_{>0} \cap N_{K / \mathbb{Q}} J_{K}}=G_{\text {loc }}
$$

in the notation of 11.3$)$. Let $P_{K}^{+}=\left\{\alpha \mathfrak{o}_{K}: \sigma(\alpha)>0 \forall \sigma: K \hookrightarrow \mathbb{R}\right\}$ be the group of totally positive principal fractional ideals of $K$. Recall that the idealtheoretic formulation of global class field theory gives an inclusion-reversing bijection between subextensions $L$ of the narrow class field of $K$ and subgroups $H$ of $I_{K}$ satisfying

$$
I_{K} \supset H \supset P_{K}^{+} \text {. }
$$

For a subextension $L$ corresponding to a subgroup $H$ as above, the Artin map gives an isomorphism

$$
I_{K} / H \rightarrow \operatorname{Gal}(L / K)
$$

We have

$$
I_{K} \supset N_{K / \mathbb{Q}}^{-1}\left(\mathbb{Q}_{>0} \cap N_{K / \mathbb{Q}} J_{K}\right) \supset N_{K / \mathbb{Q}}^{-1}\left(\mathbb{Q}_{>0} \cap N_{K / \mathbb{Q}} K^{*}\right) \supset P_{K}^{+} .
$$


Therefore, class field theory tells us that both $N_{K / \mathbb{Q}}^{-1}\left(\mathbb{Q}_{>0} \cap N_{K / \mathbb{Q}} J_{K}\right)$ and $N_{K / \mathbb{Q}}^{-1}\left(\mathbb{Q}_{>0} \cap N_{K / \mathbb{Q}} K^{*}\right)$ correspond to finite abelian subextensions of the narrow class field of $K$ which we will denote by $L_{\mathrm{loc}}$ and $L_{\text {glob }}$ respectively. We have

$$
\operatorname{Gal}\left(L_{\mathrm{loc}} / K\right) \cong \frac{I_{K}}{N_{K / \mathbb{Q}}^{-1}\left(\mathbb{Q}_{>0} \cap N_{K / \mathbb{Q}} J_{K}\right)} \cong G_{\mathrm{loc}}
$$

and

$$
\operatorname{Gal}\left(L_{\text {glob }} / K\right) \cong \frac{I_{K}}{N_{K / \mathbb{Q}}^{-1}\left(\mathbb{Q}_{>0} \cap N_{K / \mathbb{Q}} K^{*}\right)} \cong G_{\text {glob }}
$$

Observe that $G_{\text {glob }}$ surjects onto $G_{\text {loc }}$ with kernel

$$
\frac{\mathbb{Q}>0 \cap N_{K / \mathbb{Q}} J_{K}}{\mathbb{Q}_{>0} \cap N_{K / \mathbb{Q}} K^{*}}
$$

The following result shows that this kernel is equal to the knot group.

Lemma 2.3. We have

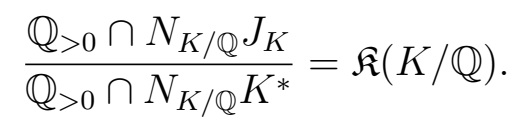

In particular, $\# G_{\text {glob }}=\# G_{\text {loc }} \cdot \# \mathfrak{K}(K / \mathbb{Q})$.

Proof. The second part of the lemma is obvious. To see the first part, we have

$$
\frac{\mathbb{Q}_{>0} \cap N_{K / \mathbb{Q}} J_{K}}{\mathbb{Q}_{>0} \cap N_{K / \mathbb{Q}} K^{*}} \hookrightarrow \mathfrak{K}(K / \mathbb{Q})
$$

with cokernel

$$
\frac{\mathbb{Q}^{*} \cap N_{K / \mathbb{Q}} J_{K}}{\left(\mathbb{Q}_{>0} \cap N_{K / \mathbb{Q}} J_{K}\right) N_{K / \mathbb{Q}} K^{*}} .
$$

We claim that this cokernel is trivial. If $K$ is totally complex then the claim follows since $\mathbb{Q}^{*} \cap N_{K / \mathbb{Q}} J_{K}=\mathbb{Q}_{>0} \cap N_{K / \mathbb{Q}} J_{K}$. Now suppose that $K$ has at least one real embedding. It suffices to show that there exists $\alpha \in K^{*}$ with $N_{K / \mathbb{Q}}(\alpha)<0$. Let us write $K=\mathbb{Q}(\beta)$ for some $\beta \in K$. Let $\sigma_{1}, \ldots, \sigma_{r}$ be the embeddings of $K$ into $\mathbb{R}$, ordered such that $\sigma_{1}(\beta)<\sigma_{2}(\beta)<\cdots<\sigma_{r}(\beta)$. Let $m$ be the number of embeddings $\sigma_{i}$ for which $\sigma_{i}(\beta)<0$. If $m$ is odd, then $N_{K / \mathbb{Q}}(\beta)<0$. If $m$ is even, choose $\epsilon \in \mathbb{Q}$ such that adding $\epsilon$ to $\beta$ changes the sign of $\sigma_{i}(\beta)$ for exactly one embedding $\sigma_{i}$. Then $N_{K / \mathbb{Q}}(\beta+\epsilon)<0$.

\section{Asymptotics For $N_{\text {loc }}(B)$ AND $N_{\text {glob }}(B)$}

Our method for estimating $N_{\text {loc }}(B)$ and $N_{\text {glob }}(B)$ is based on work of Odoni Odo73, $\S 1$ and $\S 2]$. Recall that $I_{K}$ is the group of non-zero fractional ideals of $K$. We shall proceed by proving asymptotic formulae for analogues of $N_{\text {loc }}(B)$ and $N_{\text {glob }}(B)$ in which the elements to be counted are restricted to finite index subgroups of $N_{K / \mathbb{Q}} I_{K}$. The characters of the finite quotient groups are used to 
write down explicit indicator functions which allow us to phrase the counting problems in terms of Dirichlet series. By realising the finite quotient groups in question as Galois groups of abelian subextensions of the narrow class field of $K$, we can apply Odoni's method [Odo73, §2] to relate each Dirichlet series to an Artin $L$-series in order to estimate the restricted counting functions asymptotically. Finally, we shall show how these counts can be related to $N_{\text {loc }}(B)$ and $N_{\text {glob }}(B)$ in order to establish Theorem 1.3 .

All of our indicator functions will involve the indicator function $\delta$ on $\mathbb{Q}_{>0}$ given by

$$
\delta(x)= \begin{cases}1, & \text { if } x \in N_{K / \mathbb{Q}} I_{K} \\ 0, & \text { otherwise }\end{cases}
$$

This is a multiplicative function by [Odo73, Lemma 1.1].

3.1. Local norms. The aim of this section is to provide an asymptotic formula for $N_{\text {loc }}(B)$. We begin by analysing the counting function

$$
N_{\text {loc },+}(B)=\#\left\{t \in \mathbb{Q}_{>0} \cap N_{K / \mathbb{Q}} J_{K}: H(t) \leqslant B\right\} .
$$

Using the results of $\$ 2$, together with the multiplicativity of $\delta$, we find that (cf. Odo73, Eq. (2.3)])

$$
N_{\mathrm{loc},+}(B)=\frac{1}{\# G_{\mathrm{loc}}} \sum_{\chi} \sum_{a \leqslant B} \delta(a) \chi(a) \sum_{\substack{b \leqslant B \\ \operatorname{gcd}(a, b)=1}} \delta(b) \chi(b)^{-1},
$$

where $\chi$ runs through all the characters of the finite group $G_{\text {loc }}$. Let us first consider the contribution from the trivial character $\chi=\chi_{0}$. Taking $k=a$ in Odo73, Thm. IIA] we deduce that there is a constant $c_{1}(K)>0$ such that

$$
\sum_{\substack{b \leqslant B \\ \operatorname{gcd}(a, b)=1}} \delta(b)=\frac{c_{1}(K) \lambda(a) B}{(\log B)^{1-\delta_{K}}}\left(1+O\left(\frac{1}{\log B}\right)\right)
$$

uniformly in $a$, where

$$
\lambda(a)=\prod_{p \mid a}\left(1+\frac{\delta(p)}{p}+\frac{\delta\left(p^{2}\right)}{p^{2}}+\ldots\right)^{-1} .
$$

(See [Odo73, Eq. (1.9)] for confirmation that the exponent of $\log B$ agrees with what is written here.) The contribution to $N_{\text {loc },+}(B)$ from the trivial character is therefore found to be

$$
\frac{1}{\# G_{\mathrm{loc}}} \frac{c_{1}(K) B}{(\log B)^{1-\delta_{K}}} \sum_{a \leqslant B} \delta(a) \lambda(a)\left(1+O\left(\frac{1}{\log B}\right)\right) .
$$


The function $\lambda(a)$ has constant average order of magnitude and an inspection of the proof of [Odo73, Thm. IIA] confirms that

$$
\sum_{a \leqslant B} \delta(a) \lambda(a)=\frac{c_{2}(K) B}{(\log B)^{1-\delta_{K}}}\left(1+O\left(\frac{1}{\log B}\right)\right),
$$

for an appropriate constant $c_{2}(K)>0$. Hence the trivial character makes the overall contribution

$$
\frac{1}{\# G_{\text {loc }}} \frac{c(K) B^{2}}{(\log B)^{2\left(1-\delta_{K}\right)}}\left(1+O\left(\frac{1}{\log B}\right)\right)
$$

to $N_{\text {loc },+}(B)$, where $c(K)=c_{1}(K) c_{2}(K)$.

Turning to the contribution from the non-trivial characters, an application of the triangle inequality shows that they make an overall contribution

$$
\leqslant \frac{1}{\# G_{\mathrm{loc}}} \sum_{\chi \neq \chi_{0}} \sum_{a \leqslant B} \delta(a)\left|\sum_{\substack{b \leqslant B \\ \operatorname{gcd}(a, b)=1}} \delta(b) \chi(b)^{-1}\right| .
$$

We now appeal to the proof of [Odo73, Thm. IIB], which shows that for a non-trivial character $\chi$ of $G_{\text {loc }}$ there exists a constant $e_{K}>0$ such that

$$
\left|\sum_{\substack{b \leqslant B \\ \operatorname{gcd}(a, b)=1}} \delta(b) \chi(b)^{-1}\right|=O\left(\frac{\lambda(a) B}{(\log B)^{1-\delta_{K}+e_{K}}}\right)
$$

uniformly in $a \leqslant B$. This part of Odoni's proof involves relating the Dirichlet series

$$
\sum_{b=1}^{\infty} \delta(b) \chi(b) b^{-s}
$$

to an Artin $L$-series, using the fact that $\chi$ is a character of the Galois group of an abelian subextension of the narrow class field of $K$, as shown in $\S 2$.

Combining (3.2) and (3.3) with (3.1), we conclude that

$$
N_{\mathrm{loc},+}(B)=\frac{1}{\# G_{\mathrm{loc}}} \frac{c(K) B^{2}}{(\log B)^{2\left(1-\delta_{K}\right)}}(1+o(1)) .
$$

It remains to relate $N_{\text {loc },+}(B)$ to $N_{\text {loc }}(B)$ in order to prove the first part of Theorem 1.3. If $K$ is totally complex then $N_{\text {loc }}(B)=N_{\text {loc, }+}(B)$. Now suppose that $K$ has at least one real embedding. If $-1 \in N_{K / \mathbb{Q}} J_{K}$ then $N_{\text {loc }}(B)=2 N_{\text {loc },+}(B)$. The final case to consider is therefore the case in which $K$ has at least one real embedding and $-1 \notin N_{K / \mathbb{Q}} J_{K}$.

We shall handle $N_{\text {loc }}(B)$ via the observation that the map $t \mapsto|t|$ gives a bijection of sets

$$
\left\{t \in \mathbb{Q} \cap N_{K / \mathbb{Q}} J_{K}: H(t) \leqslant B\right\} \rightarrow\left\{t \in T_{K}: H(t) \leqslant B\right\},
$$


where $T_{K}=\mathbb{Q}_{>0} \cap\left[-N_{K / \mathbb{Q}} J_{K} \cup N_{K / \mathbb{Q}} J_{K}\right]$. Thus

$$
N_{\text {loc }}(B)=\#\left\{t \in T_{K}: H(t) \leqslant B\right\} .
$$

In the proof of Lemma 2.3 , we saw that when $K$ has at least one real embedding $N_{K / \mathbb{Q}} K^{*}$ contains negative elements. When combined with our assumption that $-1 \notin N_{K / \mathbb{Q}} J_{K}$, this shows that $T_{K}$ contains $\mathbb{Q}_{>0} \cap N_{K / \mathbb{Q}} J_{K}$ as an index 2 subgroup. In particular, it follows that $G=N_{K / \mathbb{Q}} I_{K} / T_{K}$ is a finite abelian group, which by class field theory corresponds to an abelian subextension of the narrow class field of $K$. It is clear that

$$
\# G=\frac{1}{2} \# G_{\text {loc }},
$$

whence

$$
N_{\text {loc }}(B)=\frac{2}{\# G_{\text {loc }}} \sum_{\chi} \sum_{a \leqslant B} \delta(a) \chi(a) \sum_{\substack{b \leqslant B \\ \operatorname{gcd}(a, b)=1}} \delta(b) \chi(b)^{-1},
$$

where $\chi$ runs through all the characters of $G$. This can now be estimated using Odoni's argument. Once combined with (3.4), we conclude that

$$
N_{\mathrm{loc}}(B)=\frac{2^{j(K)}}{\# G_{\mathrm{loc}}} \frac{c(K) B^{2}}{(\log B)^{2\left(1-\delta_{K}\right)}}(1+o(1)),
$$

where

$$
j(K)= \begin{cases}1, & \text { if } K \text { has a real embedding, } \\ 0, & \text { if } K \text { is totally complex }\end{cases}
$$

This is satisfactory for the first part of Theorem 1.3 .

3.2. Global norms. Our starting point is an asymptotic formula for

$$
N_{\text {glob, }+}(B)=\#\left\{t \in \mathbb{Q}_{>0} \cap N_{K / \mathbb{Q}} K^{*}: H(t) \leqslant B\right\} .
$$

As before, following [Odo73, §2], we find that

$$
N_{\text {glob },+}(B)=\frac{1}{\# G_{\text {glob }}} \sum_{\chi} \sum_{a \leqslant B} \delta(a) \chi(a) \sum_{\substack{b \leqslant B \\ \operatorname{gcd}(a, b)=1}} \delta(b) \chi(b)^{-1}
$$

where now $\chi$ runs through all the characters of the finite group $G_{\text {glob }}$. The same argument used to establish (3.4) runs through as before with the outcome that

$$
N_{\text {glob },+}(B)=\frac{1}{\# G_{\text {glob }}} \frac{c(K) B^{2}}{(\log B)^{2\left(1-\delta_{K}\right)}}(1+o(1)) .
$$

Here the values of the constants $c(K)$ and $\delta_{K}$ are exactly as in (3.4). We would like to deduce from this that

$$
N_{\text {glob }}(B)=\frac{2^{j(K)}}{\# G_{\text {glob }}} \frac{c(K) B^{2}}{(\log B)^{2\left(1-\delta_{K}\right)}}(1+o(1)),
$$


where $j(K)$ is given by $(3.5)$. This will suffice for Theorem 1.3 .

If $K$ is totally complex then it is clear that $N_{\text {glob }}(B)=N_{\text {glob, }+}(B)$ and so we are done. Suppose that $K$ has at least one real embedding. If $-1 \in N_{K / \mathbb{Q}} K^{*}$ then $N_{\text {glob }}(B)=2 N_{\text {glob, }}(B)$, which is satisfactory. The remaining case to consider is therefore the case in which $K$ has at least one real embedding and $-1 \notin N_{K / \mathbb{Q}} K^{*}$. This is done in the same way as in $\$ 3.1$, using the bijection

$$
\left\{t \in N_{K / \mathbb{Q}} K^{*}: H(t) \leqslant B\right\} \rightarrow\left\{t \in U_{K}: H(t) \leqslant B\right\}, \quad t \mapsto|t|,
$$

where $U_{K}=\mathbb{Q}_{>0} \cap\left[-N_{K / \mathbb{Q}} K^{*} \cup N_{K / \mathbb{Q}} K^{*}\right]$.

\section{REFERENCES}

[Bar81a] H.-J. Bartels, Zur Arithmetik von Konjugationsklassen in algebraischen Gruppen. J. Algebra 70 (1981), 179-199.

[Bar81b] H.-J. Bartels, Zur Arithmetik von Diedergruppenerweiterungen. Math. Ann. 256 (1981), 465-473.

[Bha14] M. Bhargava, A positive proportion of plane cubics fail the Hasse principle. Submitted, 2014. (arXiv:1402.1131)

[dlBB14] R. de la Bretèche and T. D. Browning, Contre-exemples au principe de Hasse pour certains tores coflasques. J. Théor. Nombres Bordeaux 26 (2014), 25-44.

[CF67] J. W. S. Cassels and A. Fröhlich, Algebraic number theory (Proc. Instructional Conf. Brighton, 1965), Academic Press, 1967.

[CTK98] J.-L. Colliot-Thélène and B. È. Kunyavskiǐ, Groupe de Brauer non ramifié des espaces principaux homogènes de groupes linéaires. J. Ramanujan Math. Soc. 13 (1998), 37-49.

[Gur78] S. Gurak, On the Hasse norm principle. J. reine angew. Math. 299/300 (1978), $16-27$.

[HN52] G. Hochschild and T. Nakayama, Cohomology in Class Field Theory. Ann. of Math. 55 (1952), 348-366.

[Hür84] W. Hürlimann, On algebraic tori of norm type. Comment. Math. Helv. 59 (1984), 539-549.

[Hür86] W. Hürlimann, $H^{3}$ and rational points on biquadratic bicyclic norm forms. Arch. Math. (Basel) 47 (1986), 113-116.

[Lou14] D. Loughran, The number of varieties in a family which contain a rational point. Submitted, 2014. (arXiv:1310.6219).

[Man71] Y. I. Manin, Le groupe de Brauer-Grothendieck en géométrie diophantienne. Actes du Congrès International des Mathématiciens (Nice, 1970), 401-411, Gauthier-Villars, Paris, 1971.

[Neu91] J. Neukirch, Algebraic number theory. Springer-Verlag, 1991.

[NSW08] J. Neukirch A. Schmidt and K. Wingberg, Cohomology of number fields. Grund. math. Wissen. 323, Springer-Verlag, 2008.

[Odo73] R. W. K. Odoni, The Farey density of norm subgroups of global fields. I. Mathematika 20 (1973), 155-169.

[Raz77] M. J. Razar, Central and genus class fields and the Hasse norm theorem. Compositio Math. 35 (1977), 281-298.

[San81] J.-J. Sansuc, Groupe de Brauer et arithmétique des groupes algébriques linéaires sur un corps de nombres, J. reine angew. Math. 327 (1981), 12-80. 
[Ser12] J.-P Serre, Lectures on $N_{X}(p)$. CRC Press, Boca Raton, FL, 2012.

[Wei14] D. Wei, The unramified Brauer group of norm one tori. Adv. Math. 254 (2014), 642-663.

School of Mathematics, University of Bristol, Bristol, BS8 1TW, UK

E-mail address: t.d.browning@bristol.ac.uk

Max Planck Institute For Mathematics, Vivatsgasse 7, 53111 Bonn, GerMANY

E-mail address: rachel@mpim-bonn.mpg.de 